\title{
Pouvoir et amertume du fétiche.
}

Deux études de cas : les Koma du Cameroun et les Masa du Tchad

The Power and Bitterness of Fetishes, Two Case Studies: The Koma (Cameroon) and Masa (Chad)

\section{Françoise Dumas-Champion}

\section{OpenEdition} Journals

\section{Édition électronique}

URL : https://journals.openedition.org/span/1050

DOI : $10.4000 /$ span. 1050

ISSN : 2268-1558

\section{Éditeur}

École pratique des hautes études. Sciences humaines

\section{Édition imprimée}

Date de publication : 1 septembre 1987

Pagination : 141-176

ISSN : 0294-7080

\section{Référence électronique}

Françoise Dumas-Champion, "Pouvoir et amertume du fétiche. », Systèmes de pensée en Afrique noire [En ligne], 8 | 1987, mis en ligne le 07 octobre 2013, consulté le 08 décembre 2022. URL : http:// journals.openedition.org/span/1050 ; DOI : https://doi.org/10.4000/span.1050 


\section{POUVOIR ET AMERTUNE DU FETICHE}

Deux études de cas :

les Koma du Cameroun et les Masa du Tchad ${ }^{1}$

par

\section{Françoise Dumas-Champion}

Le constat que l'usage des fétiches est largement répandu de par le monde doit se tempérer aussitôt par la remarque que certaines sociétés en usent avec profusion, alors que d'autres s'y adonnent avec une prudente modération. La différence est grande, par exemple, entre les populations du Golfe de Guinée qui ont fourni prétexte au concept (les Portugais ayant forgé le terme de feitiço ${ }^{2}$ : objet artificiel, par comparaison avec les amulettes, talismans, charmes et images de saints dont ils faisaient usage) et les Nuer qui reconnaissent dans cette pratique un emprunt récent et de portée limitée 3 . On pourrait dire, en forçant le trait, qu'il existe des "sociétés à fétiches" et "sociétés sans fétiches". Ceci pour marquer une

1 Pour les langues africaines, nous avons adopté une transcription simplifiée : $\varepsilon=$ e ouvert, é : é fermé, $\eta=$ son "gn" du français, $u=$ son "ou" du français, $t=$ son "tch", g est dur (il n'a jamais le son "j"), w: "o" ouvert, $\theta$ = affriquée latérale, palato-alvéolaire.

2"Fétiche : portugais feitiço, objet, fée, maléfice; du latin facticius, qui provient d'une fabrication non naturelle" (Larousse encyclopédique).

3Evans-Pritchard, dans Nuer Religion (1956: 99) rapporte, d'après une note du Cpt Fergusson, que les iver auraient emprunté les fétiches à leurs voisins les Dinka qui, eux-mêmes, les auraient pris aux Jur vers 1902. 
différence de statut sur laquelle nous vous nous interroger. Deux expériences de terrain nous ont fait faire l'épreuve de cette différence. Chez les Koma des Monts Alantika du Cameroun, le rang politique et social d'un homme dépend des fétiches qu'il détient. Leur acquisition $n^{\prime} a$ rien d'individuel, elle n'est pas réservée, non plus, à une catégorie de prêtres, mais résulte d'un système de stades initiatiques qui engage tous les hommes de la société. Au centre d'une institution cultuelle, la nature et la fonction de ces fétiches révèlent l'organisation sociale et politique de la société koma. Les Masa du Tchad, au contraire, portent un jugement plutôt négatif sur les fétiches. Mettant l'accent, non pas sur les avantages qu'ils procurent, mais sur les inconvénients qu'ils occasionnent, ils les placent dans la catégorie de "l'amer", et ne leur confèrent aucun rôle politique et social. Ils en usent à titre privé et avec modération.

L'emploi du terme "fétiche" a été si décrié par les anthropologues que son utilisation peut paraitre aujourd'hui malvenue. Nous avons cependant pris le parti de présenter sous cette appel. lation, entendue dans sa plus large extension, toutes sortes d'objets cultuels, de végétaux ou de compositions d'éléments investis de pouvoirs spirituels, avec le souci de n'en exclure aucun a priori. Il peut s'agir de "charmes" qui confèrent prospérité, chance et santé, mais aussi d'objets sacrés dotés de propriétés magiques. L'esprit de cette recherche n'est pas de proposer une définition du "fétiche", mais de s'interroger sur cette divergence idéologique en prenant en compte les traits de l'organisation sociale de ces deux sociétés. Nous présenterons la nature du "fétiche" dans chacune d'elles en inventoriant tous les objets, emblèmes et végétaux a priori susceptibles de cette appellation, en précisant leur mode de fabrication, leur mode d'action et les implications sociales qui s'y attachent. 


\section{A - DES OBJETS CULTUELS, SYMBOLES DE POUVOIR. CAS DES KOMA DU CAMEROUN4.}

\section{Place des fétiches dans l'organisation politique de la société.}

L'institution cultuelle que nous allons considérer présente l'intérêt d'être largement diffusée parmi les populations de la Haute et Moyenne Bénoué puisqu'en dehors de son ethnie d'origine, les Samba 5 et les groupes qui lui sont apparentés, les Wom et les Mumbake, elle est attestée chez les Koma, les Véré ainsi que chez les Jukun of elle est connue sous le nom de Buhor. On trouve encore trace de ce culte dans les Grassfields en raison de l'émigration des Samba Leko qui ont fondé, au dix-neuvième siècle, les cinq chefferies de Bali Nyonga, Bali Kumbad, Bali Gham, Bali Gaso et Bali Gangsin dans la région de Bamenda.

Les populations de la Haute Bénoué qui, d'après l'historien Eldridge Mohammadou, faisaient partie de la confédération Kona, l'une des composantes, avec la confédération M'bum et Jukun, de l'empire de kororafa du $17 \mathrm{e}$ au $18 \mathrm{e}$ siècle, ont conservé des traits communs sur le plan religieux et social. Elles sont caractérisées, pour la plupart, par des traits de matrilinéarité. Mais la transmission des cultes s'effectue en ligne paternelle. A l'instar des Véré, des Bachama et des Bata, les Koma croient en un dieu céleste et lointain. Ils ne font aucun rite au soleil et $n^{\prime}$ ont aucune divinité de la terre, se distinguant par là des autres populations de la Bénoué. La vie religieuse des Koma s'organise autour d'un culte

4Les Koma qui sont répartis sur les territoires du Cameroun et du Nigéria forment une population de 3000 individus côté camerounais. Ils sont divisés entre quatre groupes autonomes comprenant d'est en ouest : les Goonu, les Gumbé, les Ritibé et les Gamé. Ils parlent trois dialectes différents. Pour une présentation générale de la population koma, se reporter à notre article (Dumas-Champion 1986). 5 Cette population est ausi désignée par le nom de Chamba. 
rendu à des ancêtres ${ }^{6}$ qui sont les intermédiaires indispensables pour communiquer avec le dieu créateur de l'univers qu'on se représente sous une forme double, masculine et féminine. Le mâle provoque le tonnerre et la pluie tandis que son épouse tend l'arc-en-ciel. C'est à eux qu'on s'adresse pour obtenir les bienfaits que l'on souhaite : récolte abondante, chasse fructueuse et nombreuse descendance. Il existe d'autres "divinités" mineures qui sont propitiées en raison des maux qu'on leur attribue spécifiquement. Les Koma les classent par catégories selon l'endroit où elles sont censées résider, arbres ou certains cours d'eau?.

En revanche, ces populations présentent des différences notables concernant l'organisation politique. Les Koma, les Véré et les Doayo ne connaissent pas de pouvoir centralisé, à l'opposé des Jukun, Bachama, Bata et Samba, organisés en chefferies. L'organisation politique des Koma apparât très proche de celle des Véré. Ces derniers, qui sont répartis en sept groupes, ne connaissent ni organisation clanique, ni union en cas de guerre, ni fonctions sociales communes. L'unité politique est le village. De même, les Koma n'ont pas de principe lignager prééminent, à l'exclusion toutefois des $G$ mé, qui sont "sambaïsés" et qui possèdent, comme leurs voisins, une organisation clanique.

${ }^{6} \mathrm{Ce}$ culte se manifeste notamment par :

- la conservation des crânes des parents, crânes transformés en objets cultuels destinés à favoriser les récoltes;

- la garde des vohté, corne dans laquelle on recueille le dernier souffle de vie d'un proche. Chaque corne, représentant l'âme du défunt, est entreposée, chez les Gımé, avec les vohté du clan. Un sacrifice accompagné de libation de bière est organisé annuellement, ou ponctuellement lorsqu' un vohté est tenu responsable d'une maladie;

- la présence de l'araignée dans les rituels, témoin indispensable et révélateur de la communication avec le monde souterrain contrôlé par les ancêtres;

- la croyance que l'enfant n'est pas un être nouveau, mais un ancêtre qui revient.

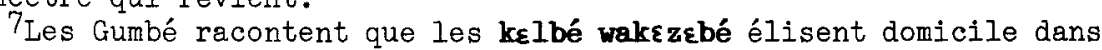
les arbres, c'est pourquoi des précautions doivent être prises au moment de l'abattage. Les kelbé mambubé affectionnent certains cours d'eau identifiables par les bruits insolites qu'on y entend: chants de coq, hennissements de chevaux...ou par les phénomènes cosmiques étranges qui s'y manifestent : des éclairs déchirant la surface de l'eau, par exemple. Il est dangereux de se baigner dans de telles rivières; on court le risque de s'y noyer. 


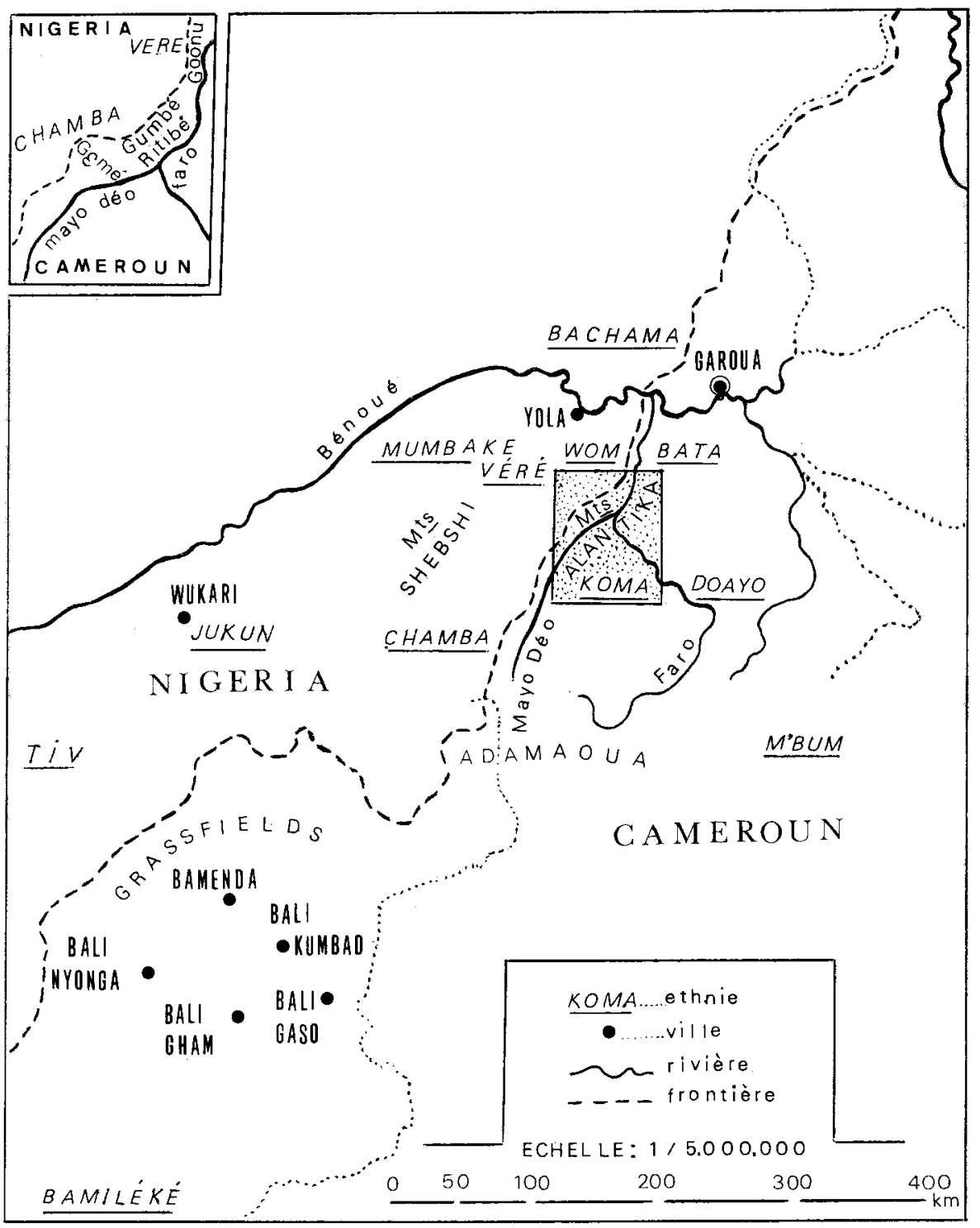

SITUATION GEOGRAPHIQUE DES KOMA. 
L'institution considérée, dénommée d'un terme générique voma, en langue samba leko ${ }^{8}$, inclut un grand nombre de cultes associés à des maladies ou à d'autres formes de malheur et dont la célébration est toujours en corrélation avec le cycle agraire. C'est sans doute pour cette raison que Meek (1931b: 348) a présenté cette institution comme un culte de pluie. En fait, il ne s'agit pas pour les Koma d'une activité cultuelle parmi d'autres - ou d'un simple culte visant à chasser une épidémie - puisque I'organisation du vomé (ou kéné) permet d'appréhender la structure politique et sociale.

Chaque communauté est dirigée par une sorte de "prêtre" ou chef rituel qui détient son pouvoir de la quantité de cultes (vomé ou kéné) dont il est propriétaire. L'héritage de cette fonction s'effectue en ligne agnatique, des collatéraux au fils du titulaire. Le prêtre est entouré d'un collège d'officiants rituels composé de huit ou dix membres choisis parmi les plus âgés de la communauté, également détenteurs de vomé (ou kéné). La possession de ces objets confère un pouvoir qui n'est pas seulement politico-religieux, mais qui est aussi divinatoire et thérapeutique. Les végétaux qui sont associés au vomé leur donnent en effet les capacités de traiter les maladies naturelles et celles qui relèvent de la sorcellerie. Autrefois, le chef rituel prenait les décisions d'ordre politique. Il se trouvait être ainsi "chef de guerre" en raison des médecines qu'il détenait. Aujourd'hui, l'administration camerounaise a nommé des chefs de village et de quartier, soustrayant le pouvoir politique aux anciens, mais le rôle des chefs administratif's - quand ils sont jeunes - se limite à la collecte de l'impôt. Ils ne s'avisent pas de s'immiscer dans les affaires internes du village encore dirigées par les aînés, détenteurs de kéné. La structure traditionnelle villageoise est d'autant mieux conservée qu'il s'agit des villages de crête inaccessibles à l'emprise du lamidat peul installé en plaine.

8Ie terme équivalent à voma est vomé chez les Gemé, kéné chez les Goonu et les Ritibé, n'dan chez les Gumbé. Le culte et ses objets cultuels sont désignés par le même terme. 
Constituant un groupe social endogame et une unité villageoise propre, les forgerons jouent un rôle prépondérant chez les Koma et parmi les populations de la Haute Bénoué. Considérés par la tradition comme les inventeurs et les pourvoyeurs des vomé ou kéné, ils incarnent, dans la mythologie tchamba et dakka9 le rôle du héros civilisateur (Frobénius, 1925/1984: 56). Personnage ambigu à qui est associée l'origine de toutes choses, traité comme un roi - notamment lors de ses funérailles - le forgeron est aussi objet d'évitement en raison des tâches dangereuses qui lui incombent: il est fossoyeur, circonciseur et sacrificateur. Il lui est interdit d'entretenir des rapports de commensalité et de promiscuité avec le reste de la population. Son activité est disjointe de celle du chef de pluie qui possède, lui aussi, à cet effet, des vomé spécifiques. On trouve, aux côtés du forgeron, des sortes de bouffons sacrés qui l'assistent lors de l'initiation (ef. Dumas-Champion 1986). Choisis parmi les adolescents qui ont montré le plus de vaillance pendant la circoncision, ils apparaissent comme les gardiens de l'ordre masculin et de la suprématie des aînés, dirigeant leur bouffonnerie et leur dérision contre les femmes et les incirconcis. Cette fonction peut être transmise de père en fils. Ils sont quelques-uns par village - autrefois semble-t-il plus nombreux quand ils accueillaient de leurs pets tonitruants les colonisateurs qui leur donnèrent le nom de "Koma-péteurs".

Toute la vie d'un homme semble orientée vers un seul but, celui d'acquérir les "choses" du vomé, sans lesquelles il ne peut être intégré à la société. La transmission du culte s'effectue en ligne paternelle, mais il ne suffit pas d'hériter pour être détenteur de voné ni pour être coopté par les officiants du village. Le secret du vomé n'est révélé qu'après une succession de stades initiatiques 10 qui jalonnent la vie d'un homme.

L'initiation à la connaissance débute avec la circoncision. Quelques jours après l'opération, les aînés vont enseigner aux

9 Nous respectons 1 'orthographe des auteurs.

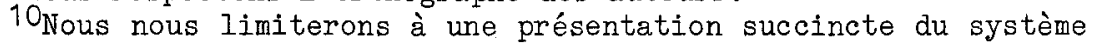
initiatique. 
jeunes circoncis le rôle des romé - non pas ceux qui sont tenus secrets mais ceux qu'ils ont déjà pu voir lors des danses précédant la circoncision, ou à l'occasion des funérailles ou encore lors de la fête agraire de vomvumé (la "bière du voné"). Il s'agit principalement de neuf flûtes en bois dites vomzié (pl.) dont ils vont apprendre ̀̀ jouer.

Comme chez les Koma, les nouveaux initiés samba et $g$ mé apprennent à tailler des rhombes en bois et à les faire tournoyer. Lors de leurs déplacements en brousse, ils signalent leur présence aux femmes avec ces rhombes et des flates. Les Gumbé, Goonu et Ritibé utilisent, quant à eux, des rhombes en fer; les initiés en prennent connaissance un an après leur circoncision; leurs pères offrent alors plusieurs pots de bière à un titulaire de kéné afin qu'il leur montre ses rhombes. Ils en apprendront le maniement et seront désormais chargés de les faire vrombir à l'occasion des cérémonies. L'initiation à la connaissance du vomé se fait individuellement ou par petits groupes de deux ou de trois, lorsque les candidats sont en mesure de payer la forte compensation ${ }^{11}$ que réclament les officiants du culte. Cette initiation, qui comporte des brimades, apparât comme le prolongement des rites de circoncision. Le postulant qui est, pour la première fois, introduit dans le bois sacré, verra certains objets du vomé, mais sans que les noms lui en soient révélés. Les jeunes gens (manglibé) 12 qui accèdent à ce stade obtiennent la garde des instruments de musique correspondant à leur rang. Il s'agit de quatre calebasses de taille différente (dites wanzi); décorées par les anciens, on les utilise comme instruments à percussion dans l'orchestre rituel. Ces jeunes hommes sont aussi préposés à siffler les kéné-golé pour annoncer la sortie des sacra au moment où ceux-ci sont régénérés. D'une manière générale, ils sont chargés des tâches matérielles de la préparation des cérémonies.

11 Le postulant doit fournir : 40 pots de bière de 15 litres chacun; une chèvre; une grande jarre de viande de boeuf cuisinée ainsi que la "boule de mil" pour accompagner le repas.

${ }^{12} C^{\prime}$ est généralement entre 15 et 20 ans qu'on accède à ce stade. Les nouveaux impétrants sont appelés manglibé (ou bengebé). Ils conservent cette appellation jusqu'au stade supérieur, quand ils deviennent nep bokep, "grands hommes". 
L'ultime stade initiatique est seulement accessible aux alnés qui sont chefs de famille. La compensation élevée ayant été fournie aux officiants, l'impétrant aura accès au savoir du vomé. C'est encore quelque temps plus tard que, moyennant une nouvelle compensation, les officiants lui remettront toutes sortes d'objets qui deviendront ses propres kéné ou vomé. Il pourra être élu par les officiants du village Iorsqu'une place sera vacante parmi eux.

Ce système à stades initiatiques confère à chaque classe d'âge une fonction rituelle spécifique. Les différentes positions occupées par les hommes du village sont mises en scène au cours de toutes les cérémonies religieuses. Cette hiérarchie s'inscrit dans I'espace territorial de la manière suivante : les circoncis sont chargés de faire vrombir les rhombes à l'entrée du village, les manglibé jouent les instruments qui leur sont réservés non loin du bosquet sacré tandis que les "grands hommes" dansent en maniant leurs kéné au sein du bois sacré. L'accès au vomé est ce qui fonde la condition d'homme. Mais il faut avoir un âge avancé et subi les différentes initiations pour être en position d'héritier ou.d'acquéreur 13 de vomé. De ce système et du principe du secret qui engage les prérogatives des initiés résulte l'institutionnalisation d'un véritable pouvoir gérontocratique.

La condition des hommes s'exprime entièrement dans cette activité cultuelle qui les distingue des femmes, cantonnées toute leur vie dans la catégorie des incirconcis. Toutefois, les femmes Gemé font exception; à l'instar des Samba, elles détiennent des objets cultuels interdits aux hommes, de la même façon que les emblèmes masculins le sont aux femmes. Des sanctions identiques (stérilité, malchance) frappent les transgresseurs, quelque soit leur sexe. Les femmes sont initiées (les fillettes de sept-huit ans subissent l'extraction des deux incisives centrales de la mâchoire supérieure, comme chez les Samba). Mais cette égalité apparente ne contrebalance pas la suprématie masculine qui s'exerce à travers les symboles religieux.

$13 \mathrm{~A}$ défaut d'héritage, les cultes sont cédés moyennant le versement d'une compensation à son propriétaire. 


\section{Nature des vomé}

Après avoir situé le rôle des vomé dans la structure sociale, il nous faut maintenant préciser l'origine et la nature de leur pouvoir. D'où viennent-ils? Sont-ils de "simples" objets cultuels? En quoi font-ils partie de la catégorie des "fétiches"? Sont-ils, par exemple, le réceptacle de divinités? L'éventail de ces sacra est très étendu. Nous le passerons en revue tout en indiquant les propriétés qui leur sont attachées.

Chaque culte se distingue par le nom qu'il porte, la fonction thérapeutique qui le qualifie et les symboles ou objets qui le représentent ; toutefois, ces symboles offrent des similitudes dans tous les cultes. Ils se répartissent en plusieurs catégories que nous avons répertoriées ainsi :

- emblèmes ;

- instruments de musique;

- vomé individuels ;

- pierres ;

- végétaux.

Les emblèmes

Arborés par les prêtres à l'occasion des grandes cérémonies telles que la circoncision, les funérailles et la fête agraire de vom vumé, ces emblèmes portent le nom de vom lamé (litt. les vomé des forgerons) bien qu'ils n'appartiennent plus exclusivement aux forgerons qui, rappelons-le, sont, d'après la tradition, les inventeurs ou les pourvoyeurs de ces symboles. Dans sa thèse consacrée aux Chamba, Fardon (1980: 52) précise que les titulaires de jubi (terme chamba daka équivalent à voma) appartiennent à un clan de forgerons qui serait d'origine Véré. Ce clan de forgerons s'est trouvé "marginalisé", puis a finalement été dépossédé de ses cultes par des patriclans chamba de Mapeo. On retrouve ces emblèmes partout ot le culte d'obédience voma est attesté et bien au-delà : notamment chez les Tiv où le chef conserve les outils du forgeron comme insignes du pouvoir (Abraham, 1968: 139) et chez les Doayo, portés par les 
bouffons (Barley, 1983: 73). Ils se composent de trois faucilles, considérées comme des instruments masculins et d'un sorte de sceptre en bois, à tête d'épervier, à caractère féminin. Chez les Goonu, les Gumbé et les Ritibé, ce dernier insigne est porté par les bouffons-péteurs qui, rappelons-le, jouent le rôle d'assistants du forgeron, lors de l'initiation masculine et féminine ou au moment des funérailles. D'autres vom

lamé : couteau de jet d'origine m'bum 14 , épées, harpon, sont déposés à l'entrée de l habitation du chef religieux, notamment le jour du départ des circoncis.

Ces emblèmes sont-ils le signe d'une fonction, ou sont-ils investis d'un pouvoir magique

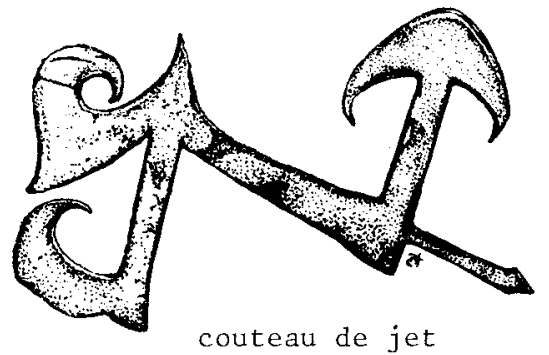
propre? A propos des Chamba, Fardon (1980: 51) rapporte qu'ils confèrent à leurs gardiens un pouvoir qui les met en relation étroite avec le monde des ancêtres.
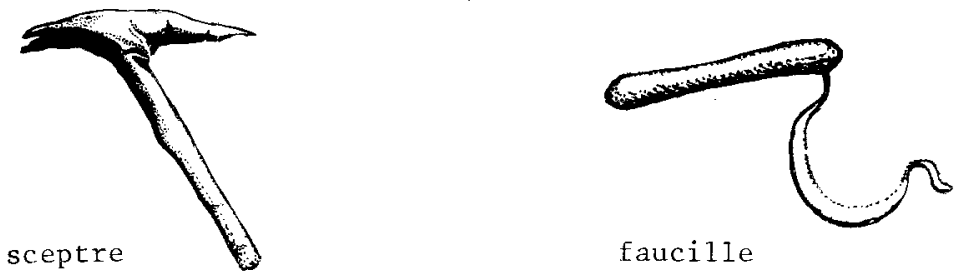

Eaucille

On dit qu'il leur donne la faculté de les voir. Le vom gara, prêtre de la communauté, peut faire trébucher un rival en décrivant une ligne avec sa faucille. Une fois à terre, celui-ci sera saisi par les esprits ancestraux habitant le monde souterrain. Ces objets

14Chez les M'bum, des couteaux de jet, de même facture, désignés par le terme ha, font partie des objets sacrés dans lesquels résident la force et la puissance du belaka (roi). Cf. à ce sujet, Froelich, 1959. Cf: aussi la reproduction photographique d'un couteau de jet m'bum dans Podlewski, 1978. Frobénius (1925/1984: 248) rapporte qu'on retrouve ce type de couteau de jet chez les Gbaya. D'autre part, Vergiat le mentionne à propos des Manja. 
sont crédités d'une telle puissance qu'il en résulte un sentiment de méfiance à l'égard de leurs détenteurss. Chez les Gemé, le harpon, vom sonné, fiché en terre, est censé communiquer ainsi avec le monde des ancêtres. Au sujet des Chamba qui ont émigré dans les Grassfields, Chilver et Kaberry (1968: 67, cité par Fardon) écrivent que "le fait d'agiter les couteaux de jet rayés de rouge et de blanc a pour effet de disperser les influences hostiles".

Ces données montrent que ces objets ne sont pas seulement l'emblème d'une fonction. Alors qu'un emblème est un signe sans action propre, ceux-ci sont dotés d'un pouvoir qui est à la disposition de leurs détenteurs, pouvoir qui tient au caractère que les esprits ancestraux impriment à ces objets.

Poursuivons la description de notre inventaire pour connâtre les propriétés des autres objets cultuels.

- Les instruments de musique.

Chaque culte dispose d'un orchestre; les instruments - dont certains sont cachés aux non-initiés, d'autres visibles lors des danses et chants collectifs - ont un champ d'action qui excède leur simple fonction musicale puisqu'ils possèdent aussi un rôle apotropaìque. Joués à chaque célébration, que ce soit à l'occasion des rites agraires ou à des fins thérapeutiques particulières, ils sont destinés à éloigner les mauvais esprits. Ces instruments sont confiés à la garde des officiants du culte. Chez les Goonu, le gardien du tambour reçoit le nom de ba-dage (père du tambour), celui qui détient les flûtes est le ba-daru. A propos des Chamba, Fardon mentionne que les chefs sont installés avec des flûtes (leera) tandis que les spécialistes des rituels, appelés wanbu, le sont avec les cornes en cous de calebasse (jub jungi), symboles par excellence du voma.

a) Les instruments à vent : Les rhombes en fer sont les premiers vomé révélés aux jeunes circoncis. Ils sont appréhendés comme la "voix des ancêtres". On dit encore qu'ils chassent les oiseaux maléfiques. Ils vont par familles : on parle des rhombes père, 
mère et enfant. Vrombis au début d'une cérémonie, ils annoncent aux femmes le moment de regagner leurs cases et leur rappellent l'interdit de regard qui les frappe.

Parallèlement au vrombissement des rhombes, retentit, au lendemain de la circoncision, le claquement d'une longue corde que les Gemé appellent "la main du vomé".

Des flûtes en cornes de gazelle et d'antilope de différentes tailles, des flutes en bois (vom zié) ${ }^{15}$ qui vont par familles de neuf, et une grande trompe (vom fossé), font également partie de I'orchestre.

Décrites par Meek comme le symbole du voma, les cornes en cous de calebasse emboîtés, qui produisent un son rauque, représentent également la voix des ancêtres. Surtout associées aux rites agraires, ces cornes sont exclusivement soufflées en saison des pluies pour favoriser la récolte à venir. Elles apportent aux femmes la preuve de l'existence du vomé qui, la nuit, vient hanter le village et ramasser les prémices de la récolte de citrouille. Durant cette période, les rhombes ne sortent pas.

On note la présence de ces cornes "en cous de calebasses", instruments-symboles du vomé, partout où le culte est attesté. La description que Meek donne de I'initiation au buhor chez les Jukun correspond trait pour trait à celle du vomé. Les propos des officiants qu'il rapporte, concernant la relation au symbole du culte, sont d'autant plus précieux qu'ils peuvent nous éclairer sur la fonction du symbole dans ce type de rituel et sur la conscience que les dignitaires en ont.

Après une série de brimades censées venir de buhor, les initiés ont désormais les yeux ouverts. L'officiant (1'Avô), procède alors au découpage d'une corne et en dispose les éléments devant le chef des néophytes. L'Avô demande : "Qu'est-ce que c'est?" Le garçon

15 Chaque trompe reçoit une appellation. La plus grande : vom zualzé désigne le "mâle" qui entonne. Les trompes qui reprennent la mélodie s'appellent sazé. Enfin, viennent les autres trompes. Les nouveaux circoncis apprendront à jouer de ces flûtes lors de leurs déplacements en brousse. 
répond : "C'est Buhor". L'Avô réplique : "Buhor! C'est donc ̧̧a Buhor? Une chose qu'on peut découper avec un couteau, tu l'appelles Buhor?" Tous les initiés répliquent : "C'est Buhor". L'Avô continue: "Qui vous a raconté ça? Comment une simple calebasse peut-elle être Buhor?" A ce moment, les autres officiants interviennent pour que cesse l'interrogatoire. Si un garçon décrivait la corne comme une calebasse, il serait mis à mort immédiatement. L'Avô s'adresse maintenant aux garcons en disant : "Ce que vous avez vu aujourd'hui ne doit jamais être révélé aux femmes. C'est le secret des hommes. Quand une personne a vu ceci, nous l'appelons un homme; et la voix des hommes doit être une." (Meek, 1931a: 370-371).

b) Les instruments à percussion : sonnailles en fer, grelots composés de deux coques de fer de forme oblongue, clochettes tubulaires ${ }^{16}$ et autres doubles cloches complètent l'orchestre cultuel. Le son métallique des grelots dit Meek (1931b: 433) à propos des Véré, représentait les étranges pas trainants des esprits des défunts. Chez les Cumbé, c'est le rôle du chef rituel d'agiter la cloche dite gelango qui annonce le rite de rougissement des kéné et des pierres, rite que nous décrirons plus loin. Les sonnailles et les clochettes qu'on agite au-dessus des autres symboles du vomé ont pour but d'expulser les sorciers et les esprits malfaisants à l'origine d'une maladie. Le rôle de cette musique apotropaïque fait penser au charivari, bien connu en Afrique lors des éclipses ou des naissances gémellaires.

16 Schweeger-Hefel (1962) reproduit les dessins de clochettes tubulaires koma parus dans Und Afrika Sprach, Bd II, Berlin, 1912-13, III: 160. Frobénius les décrit comme des plaquettes de fer oblongues et incurvées, munies d'une. bride ou d'une anse qui permet d'en réunir plusieurs sur un lacet de cuir. (fig. 21 et fig. 22 A: clochette identique chez les Dakka). Schweeger-Hefel cite encore Desplagnes (1907: 322) qui rapporte qu'on annonçait l'approche du Hogon en frappant une certaine bague, semblable à cette clochette qu'on retrouve encore chez les Konkomba du Nord-Togo, d'après Froelich in Bulletin IFAN, XI, 1949. Frobénius souligne que ces instruments servaient à chasser les gens et tout particulièrement les femmes. L'aire de diffusion de ce type de clochettes correspondrait à l'habitat des populations paléo-négritiques de l'Adamaoua (Namchi, Dakka, Paka...). 


\section{- Les vomé individuels}

Chaque membre du culte possède ses propres vomé ou kéné qu'il conserve à l'abri du regard des incirconcis dans des cases ou des greniers construits à cet effet. Ces kéné ne sont sortis qu'une fois par an, à l'occasion du rite au cours duquel ils sont régénérés. Ils forment un ensemble pour le moins hétéroclite. Parallèlement aux vomé dont il hérite, chaque impétrant qui accède au stade ultime de "grand homme" se voit remettre par tous les membres du culte différents objets collectés en brousse. Il peut s'agir d'une pierre choisie pour l'originalité de sa forme, de vieux fers de houe, de lances ou de flèches, de cornes d'antilopes, mais aussi de calebasses décorées par les anciens. On considère que l'étrangeté d'un objet le désigne à la propriété des esprits ancestraux. Aucun fétiche ne

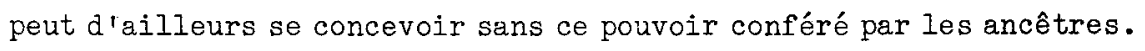
Cette pratique concernant le ramassage d'objets curieux en brousse existe aussi chez les Samba. Fardon (1981: 31) rapporte à ce sujet que "les grands coquillages ou les pierres d'une forme inusuelle devaient être rapportés au village et donnés aux aînés. Ceux-ci les examinaient et décidaient à quel culte ils devaient être ajoutés". Cette manière de procéder rappelle le premier don aux hommes d'objets métalliques, qui fut à l'origine du culte tel que les mythes le relatent. Un chef de pluie Gemé rapporte qu'autrefois les dieux et les ancêtres (vonébia)17 ont remis la puissance de la pluie aux devins dont l'âme pouvait survivre dans l'eau. (Le récit fait ici allusion à la pratique, courante en Afrique, du voyage initiatique des devins dans le monde des eaux souterraines, périple au cours duquel leur sont révélés leurs dons et leur mission). Ce pouvoir de faire la pluie est matérialisé par un vomé spécifique composé d'objets et de végétaux. Par la suite, les devins ont transmis leur pouvoir moyennant compensation. C'est ainsi que fut attribuée la fonction de chef de pluie (dite begra). Cette version corrobore les mythes samba attribuant l'origine des cultes à un ancêtre recevant une

17 Les Koma subsument divinités et ancêtres sous une même catégorie. Le terme correspondant chez les Goonu est ziule, kelbé chez les Gumbé. 
"chose secrète" (objets de fer) de la part d'un être qui se manifeste sous une apparence d'origine animale. La contrepartie de ce don est le secret. En effet, dans la légende que rapporte Fardon (1980:28), l'ancêtre qui confie ces "instruments" sans garder le secret de leur origine a été tué par la puissance donatrice qui a pris la forme du lion. Alors qu'en Afrique de l'Ouest, on attribue couramment aux petits êtres de brousse la révélation des fétiches aux hommes, les Koma qui reconnaissent également l'existence de tels êtres qui provoquent notamment des étourdissements aux individus se hasardant en brousse aux heures chaudes de la journée ou de la nuit attribuent, quant à eux, cette révélation aux dieux et aux ancêtres.

\section{4 - Les pierres du vomé}

Protectrices des cases ou des greniers qui renferment les objets cultuels, les pierres du vomé (vom béé) 18 se dressent aussi autour des bosquets sacrés où les anciens rendent leur culte. Egalement présentes devant les habitations des chefs de famille, elles jouent le rôle d'autel domestique. Drautres monolithes sont fichés au croisement des chemins menant au village ou simplement dans les champs. On trouve encore des cercles de pierres avec un monolithe érigé en leur centre, qui délimitent le "champ du vomé". Il s'agit d'une parcelle de terrain de trois mètres de diamètre qui est ensemencée par le chef religieux et les titulaires du culte. Après les semailles, on dit que "le vomé s'assoit sur cette pierre pour s'y reposer". Le rite d'intronisation

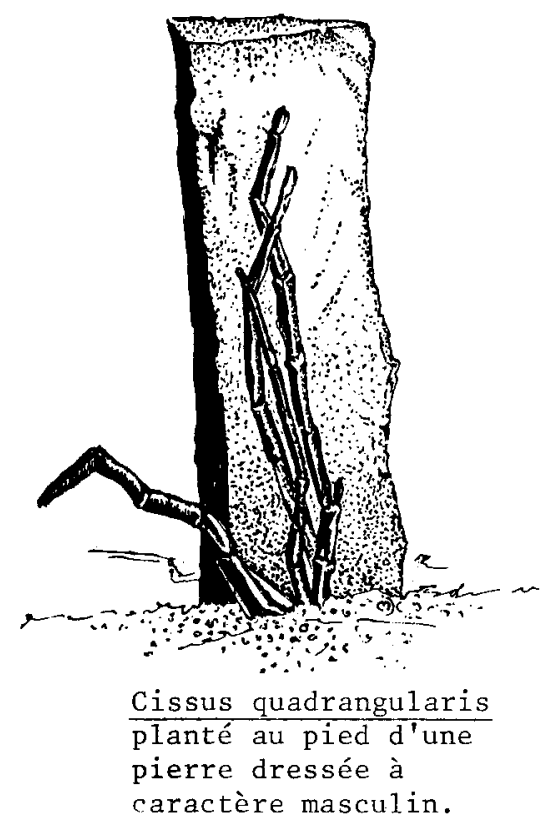

18Les Ritibé et les Gumbé désignent les pierres du kéné par le terme kéné-bité ; les Goonu par celui de kéné-bilé. 
des chefs rituels chamba consiste aussi dans le fait d'asseoir l'impétrant sur la pierre du voma. C'est un moyen pour le chef d'entrer en relation avec le monde des ancêtres. On dit d'ailleurs de ces pierres qui ne sont pas retaillées par l'homme qu'elles sont le travail des esprits ancestraux.

A la place de la pierre levée, sise à l'entrée de l'habitation, on trouve parfois un poteau en bois qui, comme les pierres, est rougi à l'ocre ou blanchi à la farine de mil. Il est habillé dans sa partie supérieure d'une touffe de feuilles. Ce pieu sacré (vom sah'lé) ${ }^{19}$, réservé au prêtre de la communauté, est le correspondant de la pierre dite "masculine" qui est accompagnée d'un monolithe "femelle", une ancienne meule dormante utilisée comme réceptacle pour les libations de la bière. Nous verrons plus loin que le traitement rituel que subissent ces pierres, en relation avec le cycle agraire, leur confère une puissance multiple qui s'exprime notamment dans leur pouvoir apotropaïque. Elles éloignent du village sorciers et mauvais esprits, à $l$ 'instar des autres vomé et interviennent dans la maturation du mil.

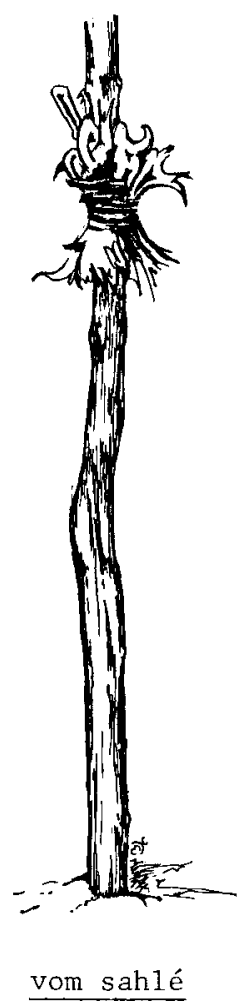

19Chez les Gumbé, ce pieu sacré est désigné par le terme saqlé. 
5 - Les végétaux du vomé

Chaque culte est associé à une série de végétaux, notamment aux feuilles d'une certaine espèce d'arbre 20 , ainsi qu'au cissus quadrangularis $^{21}$ et à aloe buttneri qui soutiennent la fonction magico-thérapeutique des autres objets du vomé que nous venons de décrire. Qualifié de goné22 mâle, en raison de ses longues tiges qui poussent au-dessus du sol, le cissus est associé à l'aloe dont l'oignon, de forme renflée et se développant sous terre, est le répondant féminin. Ces végétaux, qui sont plantés auprès des pierres, sont destinés à protéger les vomé et à renforcer le pouvoir tutélaire des monolithes. Chaque membre du culte possède, en général, plusieurs plants de cissus quadrangularis, chacun étant réservé à une fonction précise, thérapeutique ou magique. Il s'agit de protéger l'habitation et les champs, de guérir les convulsions ou de favoriser telle entreprise personnelle.

Il est intéressant de souligner la surdétermination magique et thérapeutique de la sève du cissus qui intervient dans un grand nombre de domaines. Lors du sacrifice de régénération des pierres (ou du vom sahlé) ${ }^{23}$, on écrase le cissus afin d'en extraire le

${ }^{20}$ Ces feuilles, dites vom donzé, proviennent d'un arbre au feuillage persistant qui affectionne les zones humides (arbre appelé géma chez les Gemé et girbo chez les Gumbé). En raison de ces caractéristiques, il est mis en corrélation avec la pluie et la fertilité. Comme emblèmes du vomé, ces feuilles ont divers usages. Ce sont elles, par exemple, qui enserrent le vom sahlé. Enduites d'argile et de sève de cissus, elles ajoutent une valeur de renouveau au pieu sacré réinvesti dans sa fonction tutélaire. Il est fréquent de trouver une touffe de vom donzé cachée sous une pierre barrée d'une trainée d'ocre et posée en travers de la piste qui mène au village. Cette pierre (vom wong silé) est destinée à protéger la communauté de la sorcellerie. D'autres feuilles d'un arbre appelé topé chez les Gemé sont aussi associées au vomé. (description du rituel p.160). Ce type de feuilles sert aussi à signaler qu'une naissance par les pieds vient d'avoir lieu dans un village. Les visiteurs qui s'y rendraient savent alors qu'ils ne pourront plus en sortir de la journée, selon la prescription en vigueur dans un tel cas.

21 Pour de plus amples informations, ef. Dumas-Champion 1986:52-53.

22 goné, terme générique en langue gema, désignant l'ensemble des végétaux aux vertus magico-thérapeutiques. Les Gumbé et les Ritibé emploient le terme de gané, les Goonu celui de giangu.

${ }^{23} \mathrm{Cf}$. plus loin la description du rituel. 
suc qui a la vertu de repousser les sorciers. Dans un but thérapeutique, particulièrement en cas de céphalées, on enduit de cissus les petites pierres ovales - qui accompagnent les pierres du vomé afin de s'en frotter les tempes. L'onction de ce végétal est encore largement usitée à des fins magiques : sur le bracelet du chasseur, sur son arcet ses flèches afin qu'il ne manque pas sa cible.

L'aloe buttneri couplé avec le cissus est aussi d'un usage commun. Planté à l'entrée du domicile, il est censé empêcher les serpents de pénétrer à l'intérieur de l'habitation. On le désigne sous le nom de beksx gané (fétiche contre les serpents). C'est un émétique que les Koma boivent en décoction pour soigner les céphalées et en infusion contre les diarrhées. Lors de la circoncision, on fait macérer ses tiges dans la bière de mil qu'on administre aux candidats afin de leur donner du courage. On dit aussi que le cissus leur fait perdre l'esprit. Les bouffons qui se retrouvent à côté du forgeron circonciseur absorbent ce même breuvage destiné à les soutenir dans leur tâche.

Peuple de chasseurs, les Koma ont de nombreux gané réservés à leur activité cynégétique. Ainsi le "fétiche contre la panthère", laari nabé24, qui permet au chasseur de se transformer en arbre ou en pierre au moment où le fauve attaque, comprend notamment du gané enfermé dans des cornes avec des poils de porc-épic. Le gon lété25, "l'arbre fétiche" dans lequel le chasseur dépose les cranes de ses trophées. Au pied de cet arbre pousse le goné (cissus). L'ensemble est l'objet d'un véritable culte annuel qui donne lieu à la préparation d'une bière rituelle et à l'égorgement d'un coq, sacrifice destiné à revivifier le pouvoir du fétiche et à favoriser la chasse. La manière dont meurt le volatile permet de pronostiquer la chance du chasseur (la mort brutale étant signe de malchance). L'arc, le carquois et les flèches sont arrosés du sang sacrificiel et les plumes de coq sont collées sur les armes. Tous les crânes trophées

\footnotetext{
24 Terme guma (langue parlée par les Gumbé). La panthère a disparu de la contrée depuis une dizaine d'années, mais le fétiche est encore en usage pour d'autres bêtes féroces.

25 Terme gema.
} 
sont alors enduits d'une trânée d'ocre et de suc de cissus mélangés. Ce sacrifice régénérateur est à l'image du schéma sacrificiel que nous allons analyser.

\section{Ia création des vomé}

Par quel processus les vomé acquièrent-ils le pouvoir? Nous avons vu que la matière d'un objet (le fer et la pierre) ou sa forme inusuelle le désignaient comme futur vomé, dans la mesure où ces composantes expriment la marque des esprits ancestraux et rappellent le don des sacra, tels que les mythes le rapportent.

Bien des symboles du vomé sont hérités et conservés de génération en génération. En revanche, lorsqu'un nouveau candidat se présente, les anciens du culte lui constituent un stock d'objets propre, selon les règles que nous avons énoncées. Le traitement rituel de ces simples objets les transformera en vomé. C'est dire que le rite les investit d'un pouvoir qui, sans lui, ne serait pas. La période choisie est toujours en corrélation avec le cycle agraire. L'inauguration des futurs kéné se déroulera au moment des semailles - début de la reprise des travaux champêtres - tandis que leur régénération annuelle, au cours du rite de "rougissement", aura lieu avec la fête des prémices. Les objets sont arrosés du sang sacrificiel d'un poulet avant d'être aspergés de bière. Puis, un à un, chaque objet est marqué de deux traits de terre ocre et d'un trait blanc de farine de mil germé ou de mousse blanche qui, lors du brassage de la bière, se forme à la surface du liquide. On y ajoute la sève du cissus quadrangularis (goné, gardien des vomé) qui a été préalablement écrasé. Cette onction sacrificielle, réitérée annuellement ainsi qu'à l'occasion des grands rites - circoncision, funérailles, cérémonies agraires - met en oeuvre un même schème que les Koma interprètent ainsi : la bouillie de farine de mil ou la libation de bière représentent la semence masculine, tandis que l'ocre rouge ou le sang sacrificiel symbolisent le sang féminin, principe d'ovulation. Par ces représentations associées au renouveau de la nature, les Koma mettent clairement en scène le principe de la procréation pour insuffler la vie à de simples objets et les 
doter, avec 1'aide des esprits ancestraux, d'un pouvoir capable de vaincre des éléments qu'eux-mêmes ne mâtrisent pas, comme la maladie, la sorcellerie, la sécheresse ou la stérilité. Le traitement est similaire lorsqu'il s'agit de réutiliser les outils rituels à la veille d'une cérémonie. Prenons, par exemple, la circoncision qui introduit les néophytes au premier stade de la connaissance des vomé. Le matin précédant l'ouverture des rites d'initiation, les aînés du village qui officient auprès du chef rituel procèdent au sacrifice préparatoire. Les vingt-huit couteaux de circoncision qui sont alignés à terre sont, un à un, marqués d'un trait blanc longitudinal avec un mélange de bouillie de farine de mil et de sève de cissus quadrangularis, et de deux traits d'ocre transversaux. Ainsi des rhombes, des trompes, de tous les instruments de musique et de l'ensemble des vom lamé26. Rappelons que Chilver et Kaberry, cités par Fardon (1968: 67), décrivent les couteaux de jet comme étant "rayés de rouge et de blanc". Cette marque est en effet visible sur tous les vom lamé, qu'ils soient portés, ou qu'ils soient déposés àl'entrée de l'habitation du chef rituel. C'est cette marque qui leur confère une puissance dont la nature excède le pouvoir d'un simple objet cultuel.

\section{Le pouvoir des vomé}

La création des vomé et leur régénérescence étant effectuées en relation avec le cycle agraire, le pouvoir ainsi activé et réactivé se déploie non seulement à l'égard des vomé, mais aussi sur la

${ }^{26} \mathrm{Ces}$ instruments se retrouvent à quelques détails près dans des populations avoisinantes où le culte voma n'est pas précisément attesté. Les rhombes en fer et en bois existent chez les Duru, peuple de forgerons, mais aussi chez les Lakka et chez les M'bum. Les sonnailles métalliques et les trompes également. Le même type d'offrandes leur sont faites. Frobénius (1925: 251) rapporte à propos des couteaux de jet m'bum (ha) qu'on les aspergeait de bière de mil à l'occasion des grandes cérémonies. Froelich confirme cette aspersion: "Quand les ha sont apportés au village, les vieux vont les laver à la rivière, puis se réunissent pour boire de la bière et en versent sur les ha"... (p.102) "Ils assistent aux cérémonies et dansent en tenant leurs ha à la main" (p.103). Podlewski complète l'information en les décrivant mâle et femelle et en notant qu'ils recevaient une libation de sang sacrificiel (p.110). 
production du mil. L'objet du rite est de faire intervenir les vomé dans la maturation de la céréale et de conférer de la puissance aux sacra. A ce titre, le pouvoir des vomé entre les mains des chefs rituels présente des caractéristiques similaires au pouvoir des regalia dans les monarchies sacrées.

Le pouvoir des vomé est multiforme. Apparaissant comme les gardiens de l'ordre social, ils se manifestent par différentes sortes de maux qui atteignent les non-initiés ayant transgressé l'interdit. La vue d'un vomé est le cas le plus fréquemment invoqué pour expliquer un malheur. Cet acte n'est pas toujours délibéré. Il arrive d'être involontairement en contact avec un vomé que son propriétaire a placé au-dessus d'un bien qu'il voulait protéger des voleurs.

Chaque vomé est "spécialisé" dans un type de maladie ou de malheur. Les femmes ne sont pas les seules à souffrir des vomé. Chez les Gemé, on dit que les vomé féminins (sim vomé) peuvent atteindre un homme dans tous ses modes de reproduction : sa réussite à la chasse sera affectée, son champ deviendra stérile et il connaitra l'infortune conjugale. L'origine de ces maux est révélée par le devin (le vomé n'est pas le seul émissaire du malheur. I'infortune est aussi attribuée aux proches défunts et aux petits êtres de brousse).

En cas de transgression, le traitement curatif peut recouvrir des formes très variées selon le vomé concerné et le type de maladie qu'il occasionne.

1. Le sacrifice réparateur

Le malade devra remettre au propriétaire du culte une chèvre 27 et un panier de mil qui serviront de nourritures sacrificielles pour le vomé, en échange de quoi le chef de culte demandera la guérison du patient. Les objets du vomé reçoivent le sang sacrificiel et la bière. Enfin, la viande est cuisinée puis consommée sur place

27 S'il s'agit d'un cabri trop petit pour être consommé, le chef de culte coupe l'extrémité de I'oreille pour arroser de sang les vomé, puis ramène l'animal chez lui. Il l'élèvera et le sacrifiera lorsqu'il sera adulte. Ce cabri, appelé vom bę, appartient désormais au vomé. 
par le chef de culte et ses officiants. La bière est bue convivialement. La maladie n'est pas, comme dans certaines sociétés, un appel à l'initiation.

2. Le rite destiné à tuer la puissance du vomé (vom yom nani)

Il peut s'écouler un certain temps avant que le malade ait les moyens d'offrir l'animal et le mil qui le libéreront définitivement. Le propriétaire du culte va donc tenter de dégager momentanément l'individu saisi par la puissance du vomé. Il utilise une branche feuillue provenant de l'arbre (topé), symbole du vomé. Il plonge la branche dans l'eau puis asperge tout à la fois les objets sacrés et le malade qui tourne le dos au vomé qu'il ne doit pas voir. Ce rite de bénédiction s'accompagne d'une invocation au vomé. La puissance du vomé n'est que provisoirement éteinte, bien que cette opération soit dite "tuer le vomé".

3. Une infusion thérapeutique

Tant que le sacrifice réparateur n'est pas effectué, le malade reste sous l'emprise du vomé. Le propriétaire peut être négligent et retarder la préparation de la bière. Si le vomé commence à se manifester, le malade rendra visite une nouvelle fois au chef de culte qui lui administrera une infusion du goné (cissus quadrangularis) qui protège son vomé.

4. Le traitement d'extraction d'une maladie

Le devin-thérapeute (geza) ou le chef de culte se tient debout face au malade qui est assis, jambes tendues. De l'index et du pouce préalablement induits de vom goné (ici le cissus) l'officiant presse les tempes du malade tout en invoquant l'ancêtre ou le vomé responsable de la maladie, puis, des deux mains, il presse le goné sur le corps du malade d'un mouvement qui va de la poitrine aux orteils et qui s'achève en un geste d'expulsion qui opère symboliquement l'extraction du mal. 
B - LE FETICHE AMER - CAS DES MASA DU TCHAD

\section{Statut des spécialistes dans la société}

Chez les Masa du Tchad, la mâtrise du pouvoir politique et religieux n'est pas associée à des objets sacrés. La première distinction notable, par rapport à la société koma, est cette absence de sacra ou de toute autre forme d'objet fétiche. Les Masa n'ont recours, en effet, qu'à des végétaux, utilisés comme charmes, sortilèges, philtres d'amour et remèdes. La seconde différence réside dans l'existence de spécialistes appelés sum-sa-guna (de sum-sa, les hommes et guna, terme désignant toute plante ou matière végétale dotée de propriétés magiques et thérapeutiques) 28 . Ces spécialistes tiennent leur pouvoir d'une révélation. L'élu est, en effet, possédé par les divinités qui investissent son corps et l'attirent en brousse où elles séjournent. On dit alors d'un tel individu qu'il a les fuliana (les divinités) ou les "choses" dans la tête, Oé-ka-la-mu. Tous les personnages investis d'une fonction religieuse : prêtre de la terre (bum nagata), devins (sa-ma-grayna et sa-ma-patna), propriétaire de mare (bum golonga) représentant d'un culte lié à la fertilité du sol (bum diniatta), et détenteur d'un pouvoir de guérir ou de rendre malade (bum fulla) ont été sujets à un investissement analogue. Cette prise de corps qui sanctionne le choix des divinités est la condition indispensable à la révélation. Après une période de possession intense, la divinité relâche son emprise et la communication s'instaure par voie onirique. Mulla, le sa-ma-guna de Kumi-Idik, nous rapporte comment Matna, la puissance responsable de la mort, lui indiqua en rêve la plante qu'il devait déterrer et son usage. De la même manière, Mollo, le sa-ma-guna de Baha-Walyasu, raconte comment, alors qu'il était encore adolescent et qu'il gardait le bétail, les fuliana lui avaient signalé les branches supérieures d'un arbre, (un balanites aegyptiaca) sur lesquelles poussait un

${ }^{28}$ Cette spécialité n'est pas exclusivement réservée aux hommes. De même qu'il existe des devineresses, il existe des Cada-guna (Cada signifie femme). 
oignon sauvage (aloe buttnerí) dit tayna qui, généralement, crốt enfoui dans le sol. Rapportant ce tayna chez lui, il s'enrichit rapidement et put se marier. Le tayna que Lawna, le "père des hommes", lui avait donné, lui prédisait en effet l'avenir et lui indiquait les moyens de s'enrichir. Par la suite, Mollo fit l'acquisition de trois autres guna auprès d'un baguirmien. L'un était destiné à tuer, l'autre guérissait, le troisième enfin le protégeait des autres fétiches.

Tout porterait à croire que ces "élus des dieux" soient des privilégiés et que leur pouvoir leur apporte richesse et notoriété, comme c'est le cas chez les Koma. Il n'en est rien. Le statut de sa-ma-guna apparaît, au contraire, peu enviable. En petit nombre chez les Masa-Gumay29 (un ou deux par canton), ces quelques spécialistes ne semblent pas tirer un profit notable de leur fonction. On constate qu'ils sont souvent plus pauvres que la moyenne de la population, vivant généralement seuls, sans épouses, sans parents et ayant une faible descendance, parfois même sans enfant. Le sa-ma-guna de Kumi, par exemple, a perdu toute sa famille. Il en est réduit à vivre chez un cousin éloigné, alors qu'à son âge, un homme est généralement à la tête d'une famille étendue. Le cas de Mollo est tout aussi démonstratif. Après avoir connu des années de prospérité et épousé neuf femmes, signe d'une richesse conséquente, il est aujourd'hui sans épouse - et lépreux de surcroît.

La maladie et le dénuement extrême qui frappent l'individu faisant un usage inconsidéré des fétiches, manifestent le côté dangereux de ce pouvoir qu'on dit "amer". Cette épithète qualifie une force qui poursuit sa victime jusqu'à destruction de son intégrité physique ou de sa lignée. On attribue au chien, par exemple, le qualificatif d'animal au "sang amer". A I'instar de l'homme, il est doté d'un pouvoir vengeur, tokora, susceptible de se retourner contre l'individu qui le mettrait à mort. D'autres animaux sont dits "amers", comme le margouillat et le crapaud, en raison du pouvoir

${ }^{29}$ Le groupe masa le plus important, situé au nord de Bongor, est composé d'environ 30000 individus, auprès duquel nous avons mené plusieurs enquêtes de terrain de 1973 à 1985. 
mortel qu'on attribue à leur morsure. Comme preuve de l'amertume du guna, il nous a été rapportée l'histoire suivante à propos d'un homme qui possédait chez lui une grande quantité de fétiches. Cet homme fut victime d'un incendie qui détruisit entièrement son enclos. Ses femmes ayant péri dans les flammes, seuls ses guna, enfermés dans une poterie, sont demeurés intacts. Le contraste entre la mort des épouses et la conservation des fétiches révèle que $1^{\prime}$ origine de l'incendie est imputable au nombre excessif de guna. Il est, en effet, tout à fait aisé de sortir d'une habitation masa et d'échapper aux flammes ; il est, en revanche, plutôt improbable, aux dires des Masa, que de simples matières végétales, même protégées par une poterie, puissent rester intactes après un tel sinistre.

Pourquoi le pouvoir des guna est-il finalement contraire à leur utilisateur? Si l'effet escompté s'avère bénéfique dans un premier temps, l'effet maléfique ne tarde pas à se manifester. clest un fait connu dans toutes les sociétés que le pouvoir du "fétiche" peut se retourner contre son propriétaire ou sa famille. Dans les "sociétés à fétiches", comme chez les Koma, ce retournement est minimisé. En revanche, dans les "sociétés sans fétiches" (i.e. où le fétiche ne reçoit pas de valorisation sociale), comme chez les Nuer ou chez les Masa, l'accent est mis sur le danger que comportent ces objets magiques. Ainsi, chez les Masa, un chasseur ou un pêcheur qui fait usage d'un guna 30 doit le faire avec modération, pour nourrir sa famille et non pour s'enrichir. Les fuliana (divinités), qui sont les pourvoyeurs de guna, sanctionnent ceux qui en tirent un profit abusif. Le génie de l'eau, Mununda, protecteur des poissons, frappe le pêcheur peu scrupuleux. Il pourra faire une pêche abondante, mais ses vaches crèveront ou bien les siens tomberont malades. Il en va de même pour le génie de la brousse, Bagawna, qui protège les bêtes sauvages. Les fuliana apparaissent ainsi comme les régisseurs d'un équilibre à préserver entre les hommes et les animaux.

30II s'agit du Gu-kulufna (guna + kulufna: poisson) dit encore : gu-dongoleyna (dongoleyna désigne la marmite pour la sauce), le fétiche pour avoir la sauce - généralement à base de poisson. Ce fétiches, qu'on attache au filet ou à l'outil de pêche, est composé d'une branche de gui, genre tapinathus, dit giyamna. 


\section{Nature spécifique des guna}

Malgré les dangers qu'ils présentent, les guna ont un champ d'application très large. Il semble qu'on y ait recours pour chaque événement de la vie; qu'il s'agisse de réussir dans une entreprise d'ordre matériel (pêche ou chasse), dans une entreprise spécifique, honnête ou non, de s'attirer les faveurs d'une personne ou d'engager une action maléfique. Il existe aussi des guna pour se protéger des voleurs, des sorciers ou des morts, pour mettre en échec une ordalie ou pour protéger les guerriers des armes de l'adversaire. Nous avons fait référence, chez les Koma, à des fétiches qui avaient le pouvoir de transformer un individu en pierre, lui permettant d'échapper à l'attaque d'un fauve. On entend des récits analogues chez les Masa, décrivant comment un individu a pu marcher sur l'eau ou se transformer en animal. Mais ces exploits extraordinaires sont le fait de personnalités hors du commun. Ils ne relèvent pas, précise-t-on, de l'efficace d'un guna qui serait à portée de tous. Néanmoins, certains fétiches sont connus pour exercer un pouvoir sur la nature tout aussi surprenant. Il existe notamment un guna destiné à protéger le guerrier des coups de sagaie ou des coups de bâton. En avalant une poudre composée d'un mélange de tige de mil, de l'écorce d'un arbre de type piliosligana, et d'autres ingrédients tenus secrets, ou encore en portant cette même composition en amulette autour du cou ou autour des reins, l'adversaire qui s'apprête à frapper un combattant ainsi bardé de guna verra son bâton se transformer en sciure comme s'il était subitement dévoré par les termites.

Bien que les guna soient utilisés dans les domaines les plus divers, on est frappé par l'uniformité et la simplicité de leur composition: un petit nombre de végétaux qui constituent par ailleurs la base de la pharmacopée.

1 - Le cissus quadrangularis A. Linné.

Largement usité chez les Koma, il est également l'une des plantes mấtresses chez les Masa. Cette Ampélidée, originaire de l'Inde et de Malaisie, est bien connue en Afrique centrale dans la zone des savanes. Surtout reconnue pour ses propriétés thérapeu- 
tiques, elle apparaît chez les Masa comme une véritable panacée : l'écorce sert de vulnéraire pour guérir les plaies et notamment les blessures provoquées par une arme (il s'agit du gu-kawina, de gu, radical du substantif guna, et kawina: fer, terme désignant les armes). Les racines charnues sont employées comme topique maturatif pour hâter la suppuration d'un abcès ou d'une tumeur provoqués, dit-on, par un ennemi envieux; ce remède est appelé gu-sulukna (sulukna connote l'idée d'envie). Les tiges, pilées et mélangées à de la bouillie de mil rouge, sont utilisées comme remède contre les maux de ventre féminins et les maladies des intestins (gu-Coldona). La même préparation, augmentée de tamarin constitue une médicine contre les maladies vénériennes (gu-bobona).

Les Masa cultivent cinq variétés de cissus quadrangularis, toutes appelées subulla. Celles qui sont toxiques sont généralement employées en cataplasmes, les autres en infusion ou macération.

Si le cissus est largement utilisé pour ses vertus médicinales, il l'est aussi pour son pouvoir magique qui lui permet de repousser l'âme d'un mort ou l'âme vengeresse d'un assassiné. A la mort du conjoint, le veuf ou les veuves doivent se préserver de la force dangereuse (dite yawna) qui perdure au-delà de la mort et qui émane du lien créé par les relations conjugales. Le yawna se manifeste notamment par le désir du défunt d'entraîner son conjoint dans la mort, non pas, pense-t-on, dans un but maléfique, mais parce qu'il ne peut supporter cette séparation. Afin de ne pas être emporté par le mort, les veuves attachent à leur cheville gauche un guma-yawna à base de cissus quadrangularis. Le guma-yawna n'est qu'un élément mineur du rite de séparation 31 destiné à couper tous les liens unissant l'ancien couple. Au même titre qu'une simple amulette, il est considéré assurer une protection supplémentaire, mais son seul pouvoir serait insuffisant pour repousser le yawna du défunt.

De même, un meurtrier se protégera de la vengeance post-mortem de sa victime à l'aide du guma-tokora composé de tiges de cissus quadrangularis. C'est, sans doute, en raison de ses propriétés rubéfiantes que cette plante est qualifiée d'amère et qu'elle est

${ }^{31}$ Pour la description de ce rituel, cf. Dumas-Champion, 1983: 232-233. 
censée agir comme répulsif contre le tokora. Le tueur la porte à sa bouche et s'en frictionne le corps afin de s'en imprégner. Il porte des tiges de cissus nouées autour de la tête, du cou et des poignets. De plus, il entretiendra une fumigation de cette même plante pendant sa période de réclusion. Encore une fois, le gumatokora n'est qu'un élément parmi d'autres dans l'arsenal utilisé pour se protéger du pouvoir maléfique de la victime. L'emprise du tokora ne se relâche jamais et, toute sa vie, le tueur portera sur lui des guna protecteurs. Il plantera notamment un cissus dans son champ.

2 - I'aloe buttneri A. Berger.

Associé chez les Koma au cissus quadrangularis dont il est le répondant féminin, l'aloe buttneri tient également chez les Masa une place privilégiée. Cette liliacée, très répandue dans les savanes d'Afrique occidentale et centrale, est aussi connue pour ses vertus magico-thérapeutiques.

Chez les Masa, I'aloe buttneri, appelé tayna, joue un rôle peut-être plus important que celui du cissus, car il apparât toujours dans les récits étiologiques comme le type de guna que les humains regoivent des divinités. C'est, en effet, le tayna qui est la plantefétiche de l'initiation masculine (labana). C'est encore le tayna qui symbolise les rites de fertilité de Diniatta32. Et, c'est souvent ce même végétal que le sa-ma-guna reçoit des puissances qui l'investissent dans sa fonction. A ce titre, l'aloe buttneri semble jouer le rôle de sacra, bien qu'il s'agisse ici d'une plante et non d'un objet durable. Les Masa constatent que, par opposition au caractère éphémère des végétaux, l'aloe buttneri est particulièrement vivace et que, lorsqu'il disparaît, comme cela est arrivé après la grande inondation de 1971 en pays gumay, il réapparâ̂t quelques années

${ }^{32}$ On raconte que le premier bum Diniatta ("père de Diniatta") à avoir reçu le tayna l'a partagé entre tous les prêtres de la terre. Chacun a planté un morceau de la tige de cet oignon avec un aloe buttneri quelconque. Depuis, lors des semailles, les prêtres de la terre déposent dans la calebasse aux semences quelques tiges de cet oignon, dans le but de favoriser la germination. C'est aussi dans le but d'accroftre la moisson que les chefs de famille, ou leur première épouse, déposent, avant la récolte, un morceau de tayna sur l'aire aménagée au milieu du champ. 
plus tard. Outre cette perennité qui l'apparente aux autres sacra, les Masa insistent davantage encore sur le pouvoir de féconditéque confère ce végétal. La forme renflée de son bulbe qui perdure enfoui dans le sol en saison sèche et dont la renaissance semble induire le renouveau de la nature témoigne de ce pouvoir.

Outre ce rôle, les vertus conférées au tayna sont multiples. Il entre dans la composition d'un grand nombre de guna. En voici quelques exemples.

a) Les Masa prêtent à l'aloe buttneri des propriétés galactogènes, propriétés généralement attribuées au cissus quadrangularis. Les tiges de l'oignon sont données en nourriture aux vaches, mélangées à des épis de mil blanc, en guise de gwa-mira (gwa renvoie à guna par euphonie; mira signifie lait).

b) Deux sortes de philtres d'amour sont à base de tayna : le gwa-mi $\theta$ ekna (miӨ'ekna : l'étoile) qui se transmet de mère à fille. et le gwa-tinanana33 (tinapanacoller très fortement); les amants les prennent en se jurant fidélité jusqu'à la mort.

c) Un fétiche de protection, à base de tayna séché, pour assurer la paix de l'enclos familial. Chaque membre de. la maisonnée porte à sa bouche le guma-Géena ( $\theta$ ééna: la frấcheur, la paix) en guise de purification (porra) pour préserver la paix.

d) le gwa-nalina (galina: pot à sauce) est censé purifier les ustensiles de cuisine appartenant à l'épouse qui aurait proféré des jurons à l'encontre de son mari, ou qui, pour se défendre, l'aurait mordu. L'épouse, s'étant procuré auprès du sa-ma-guna un morceau de tige de tayna en frottera tous ses ustensiles de cuisine afin d'en lever la souillure, faute de quoi le mari périrait.

\section{3 - Le gui, genre tapinathus}

Surtout connu comme moyen d'obtenir des pêches fructueuses, les propriétés attribuées à ce végétal, appelé giyama (cf. note 29), varient selon l'espèce d'arbre qu'il parasite et selon les indications des fuliana, transmises par voie onirique. Cueilli sur un cailcédrat, on l'utilise, en raison de l'amertume de cet arbre,

${ }^{33} \mathrm{Cf}$. plus loin pour la composition de ce guna. 
comme protection contre les sorciers; cueilli sur un nurlona, il est utilisé comme philtre d'amour.

Il est assez difficile de décrire la pharmacopée précise des guna, car leur pouvoir résulte aussi de la relation qui se trame entre les puissances donatrices et le bénéficiaire. Il faut donc prendre en compte l'histoire individuelle de chaque sa-ma-guna. Ainsi, alors que le guma-tokora est généralement à base de cissus, certains spécialistes en fabriquent à base de tayna, ces deux végétaux pouvant être interchangeables. Les règles ne sont donc pas immuables.

Les autres traits caractéristiques du guna exploitent un rapport d'homéopathie et de contiguìté avec le but recherché. Ainsi, pour retrouver la piste du bétail volé, on fabrique un guna à partir de la terre où sont marquées les empreintes des vaches. Pour conférer à un homme une force colossale, on lui administre une préparation à base de fourmis, dite gu-orbeyda (orbeyda: fourmi), la fourmi étant capable de transporter des charges énormes, proportionnellement à son corps; ce guna est censé permettre au voleur de transporter une vache sur son dos ce qui lui permet d'accomplir son acte sans laisser d'empreinte. De même, le philtre d'amour gwa tinanana, destiné à sceller l'union des amants, comprend l'écorce d'un arbre dont les écailles se recouvrent deux à deux, ainsi que des insectes qui parasitent la capsule du coton et qui ont la particularité d'être toujours deux à deux, "collés ensemble" dit-on. Ces ingréđients sont pilés avec un morceau de tayna pour être mêlés à la nourriture.

\section{Mode d'acquisition et fabrication des guna}

La quête des plantes-fétiches est, nous l'avons vu, d'inspiration divine. Les puissances désignent à quelques privilégiés le lieu où le végétal doit être déterré et leur indiquent l'usage auquel il est réservé. La manière dont le spécialiste traite ses plantes montre qu'il n'en est pas le propriétaire, mais plutôt un gardien qui invoque la puissance tutélaire à chaque manipulation. La prière prononcée par Mollo avant de composer un fétiche abonde dans ce sens : "Lawna ! toi qui m'as donné des guna, il faut que tu m'indiques l'écorce avec laquelle je dois guérir les gens". 
Demeurant sous l'entière dépendance des fuliana, les spécialistes doivent entretenir de bonnes relations avec ces puissances; ce qui nécessite une activité sacrificielle soutenue. A défaut, 1 "amertume" des guna se manifesterait. Le prélèvement d'une parcelle de la plantefétiche est toujours précédé d'un sacrifice destiné à protéger le spécialiste. La plante est arrosée du sang d'un poulet au bénéfice de la puissance qui, en retour, laisse l'utilisateur opérer sans dommage.

Les spécialistes ne créent pas les guna. Mais, bénéficiant de la nature propre de ces fétiches qui sont tirés du règne végétal, ils ont la possibilité de les multiplier et de fournir ainsi les acquéreurs potentiels. Tous les chefs de famille possèdent des guna pour la garde de la maisonnée. Ils ont planté à l'intérieur de l'enclos, ou dans les champs, plusieurs variétés de cissus quadrangularis et de aloe buttneri dont ils gardent l'usage secret. Les individus atteints par les maléfices d'un guna deviennent à leur tour propriétaires du guna utilisé en thérapie. Par la suite, on pourra les consulter pour cette maladie précise. La transmission des guna est héréditaire. Le fils hérite des fétiches de son père, la mère transmet les siens à sa fillle aînée. Un père ne révèle l'endroit où sont enterrés ses guna et leur usage qu'au moment ou il voit sa mort venir. L'acquisition d'un guna auprès d'un spécialiste ne se limite pas à la matière végétale, elle nécessite aussi la transmission du savoir religieux qui permet d'entretenir le fétiche. C'est dire qu'on doit respecter les rites sacrificiels qui accompagnent chaque opération.

La multiplication des guna dépend évidemment des contraintes horticoles. Ainsi, on peut remettre à un acquéreur un bulbe d'aloe buttneri provenant du pied-mère. Il est doté du même pouvoir que l'oignon d'origine. S'il s'agit d'un cissus, une bouture fera l'affaire. On peut encore, comme le rapporte le récit sur la prolifération de tayna de Diniatta (cf. note 32), donner un morceau de l'oignon que le récipiendaire doit planter au contact d'un oignon ordinaire, lequel, en vertu du principe de contiguïté, se transformera en guna. En général, il suffit de prélever une parcelle du végétal pour bénéficier de toute la puissance du fétiche. Ici, comme bien 
souvent, la partie vaut pour le tout (le terme guna s'applique d'ailleurs autant à la parcelle du végétal qu'à la plante considérée dans son entier). Lorsqu'on plante un guna, aucun sacrifice n'accompagne l'opération. Les seuls rites sacrificiels ont lieu annuellement lors de la régénération de la plante et à l'occasion d'un prélèvement. C'est que, chez les Masa, ce n'est pas le rite qui transforme le végétal en fétiche. La plante possède les caractéristiques du guna si les fuliana l'ont désigné comme tel. La régénération sacrificielle d'un guna est placée sous le contrôle des fuliana et le guna n'est qu'une vulgaire plante si les fuliana ne lui confèrent pas sa force.

\section{Conclusion}

La distinction que nous avons opérée en introduction entre "sociétés à fétiches" et "sociétés sans fétiches" visait seulement à marquer une différence de valorisation. En effet, il apparaît difficile de croire qu ' une société traditionnelle puisse faire l'économie de ces moyens d'action magico-religieux que sont les fétiches. Il demeure néanmoins que des peuples comme les Nuer ou les Dinka d'après Evans-Pritchard et Lienhardt - ignoraient ces "objets" au début du siècle et que, malgré l'emprunt qu'ils en ont fait, ils éprouvent à leur égard la plus vive appréhension. Par ailleurs, les Masa avouent facilement leur incompétence en la matière, reconnaissant volontiers cette habileté à leurs voisins mundang. Ils insistent sur I'amertume du fétiche, dont la possession reste une affaire privée. A l'opposé, il y a dans la société koma une fonction du fétiche qui fait passer outre aux dangers que son utilisation comporte.

Si nous avons nommé "fétiches" les voné et les guna, c'est parce que ce terme nous a semblé le moins inapproprié pour rendre compte, en dépit d'évidentes disparités, d'une certaine communauté de nature, autorisant à comparer deux sociétés qui adoptent des attitudes différentes, à l'égard des sacra, charmes, objets et végétaux inventoriés.

Tant chez les Koma que chez les Masa, le pouvoir des fétiches ne se résume pas à celui de simples objets cultuels. On leur attribue des propriétés magiques pouvant être utilisées aussi bien dans un 
sens bénéfique - pour guérir, apporter la prospérité, favoriser la récolte - que dans un sens maléfique, puisqu'ils sont à même d'inoculer une maladie pouvant entraîner la mort. Mais, à la différence des guna masa auxquels un individu recourt pour son propre compte, les vomé agissent comme des régulateurs sociaux. On pourrait appliquer aux vomé (ou kené) des Koma, la définition qu'un informateur m'bum donne des ha : "c'est à la fois le fétiche et la chefferie" (Froelich, 1959: 104). En effet, c'est bien dans le pouvoir des vomé que réside la force de la communauté. Mais, bien plus qu'un support matériel de vie, les vomé institutionnalisent la différence des sexes et la hiérarchie entre les classes d'âge. Ils représentent l'ordre et les maux qu'ils envoient viennent sanctionner les transgressions des règles sociales.

En tant que don des dieux, les fétiches nous semblent intégrés dans les deux cas au domaine religieux. L'objet ou le support végétal n'est rien par lui-même. Il tient sa puissance du monde des ancêtres chez les Koma et directement des divinités chez les Masa. A ce titre, l'usage polyvalent d'un petit nombre de plantes pour un large éventail d'utilisations thérapeutiques montre le caractère déterminant de la relation entre le spécialiste et l'entité spirituelle donatrice.

Ils nous apparaissent aussi individualisés et distincts les uns des autres qu'un homme peut l'être de son voisin. Chaque vomé (ou kéné) est unique. On connaît son origine. Il possède un nom qui lui est propre, même s'il fait partie d'une catégorie d'objets qui composent habituellement tous les cultes vomé. Cette valeur se retrouve chez les Masa. Lorsque meurt, par exemple, le tayna que le sa-ma-guna Mollo a reçu de Lawna, il en est définitivement dépossédé. Cette conception ne va pas à l'encontre du principe de reproduction du tayna de Diyiatta qui s'apparente à un engendrement. Le tayna que possède chaque chef de terre se rattache au tayna originel à la façon dont un enfant dérive et se distingue de ses parents.

Les fétiches appartiennent au monde du secret. Chez les Masa, le savoir des guna est réservé à quelques spécialistes. Chez les Koma, l'acquisition des vomé (ou kéné) repose sur un savoir initiatique réservé aux hommes et à la classe d'âge des aînés. Cette connaissance doit être tenue secrète et les objets-fétiches, pour 
la plupart, ne doivent pas être vus - les vomé les plus puissants étant soustraits au regard des non-initiés. Leur manifestation sonore, en revanche, joue un rôle primordial, puisqu'une partie des vomé sont des instruments de musique et que le charivari auquel ils donnent parfois lieu est un mode d'action pour chasser les sorciers. S'il est interdit aux femmes de voir les rhombes, il y a comme une prescription à ce qu'elles les entendent. Leur connaissance doit être auditive. On se charge de leur indiquer la signification de ce vrombissement qui manifeste l'existence du vomé. L'importance du fétiche s'exprime dans cet interdit qu'il fait peser sur les femmes. Symbole de l'ordre social, il marque la séparation des sexes. I'interdit de voir suggère une autre interrogation. Existe-t-il parce que la vision qu'offre l'oeil ne donne pas une juste représentation de la réalité ? Le prêtre du buhor ne s'interroge-t-il pas ainsi : "Comment une simple calebasse peut-elle être Buhor ?" La véritable essence du vomé relève du domaine de l'invisible.

Ils occupent enfin une position stratégique dans l'organisation de la société traditionnelle où ils traduisent un besoin de puissance et de légitimation dans l'ordre de l'occulte. En termes de sociologie politique, on peut estimer qu'ils résultent d'une réification des relations sociales car les principes de structuration de la société y apparaissent soustraits à la connaissance de ceux sur qui elle s'exerce. Ce recours n'en est pas moins ambigu du fait qu'il place les hommes sous la dépendance des forces qu'ils sollicitent.

Les caractéristiques que nous venons d'énumérer nous permettentelles de proposer une définition du fétiche? Nous n'irons pas jusqu'à l'affirmer car celle-ci ne pourrait être appliquée à de nombreux objets pouvant aussi bien prétendre au même nom; comme c'est le cas, par exemple, des bo des Evhé, dont l'efficacité, s'il faut en croire A. de Surgy, ne dépend très souvent du bon vouloir d'aucune divinité, d'aucun ancêtre, ni même d'aucun esprit errant. Cette imprécision nous incline à douter de la valeur conceptuelle d'un terme qu'il est difficile de séparer de l'intention de dévalorisation qui est à son origine.

Françoise Dumas-Champion

Laboratoire associé $n^{\circ} 221$

CNRS - EPHE 
Ouvrages cités

Abraham R.C.

1968 The Tiv people, Farnborough, Gregg Press.

Barley N.

1983 Symbolic structures. An exploration of the culture of the Dowayos, Cambridge, University Press et Paris, Editions de la Maison des Sciences de l'Homme.

Chilver E.M. et Kaberry P.M.

1968 Traditional Bamenda. Ministry of Primary Education \& Social Welfare and West Cameroons Antiquities Commission, Cameroon.

Desplagnes I.

1907 Le plateau central nigérien, Paris, Larose.

Dumas-Champion F.

1983 Les Masa du Tchad. Bétail et société. Cambridge, University Press et Paris, Editions de la Maison des Sciences de I'Homme.

1986 "Pierres de vie", in 1'Univers du Vivant 8, 48-61

Evans-Pritchard E.E.

1956 Nuer religion, Oxford, Clarendon Press.

Fardon R.0.

1981 The Chamba: a comparative history of tribal politics, P.H.D., London, University College.

Frobénius L.

1925 Dichten und Denken im Sudan, t. V, Atlantis. Trad. Eldridge Mohammadou, Centre de recherches et d'études anthropologiques, Garoua, 1984 .

Froelich J.C.

1959 "Notes sur les M'boum du Nord-Cameroun", in Journal de la Société des Africanistes, t. 29, 1,91-117.

Meek C.K.

1931a A Sudanese Kingdom, London, Kegan Paul, Trench, Trubner and $\mathrm{C}^{\circ}$, Ltd.

1931b Tribal studies in Northern Nigeria, 2 vol., London, Kegan Paul, Trench, Trubner and $\mathrm{C}^{\circ}$, Ltd.

Mohammadou E.

1978 Les royaumes foulbé du plateau de 1'Adamaoua au XIXe siècle, Tokyo, I.L.C.A.A.

Podlewski A.

1978 "Notes sur les objets sacrés traditionnels Mbum", in Journal des Africanistes, t.48, 2, 102-120.

Schweeger-Hefel A.M.

1962 "Les insignes royaux des Kurumba", in Journal de la Société des Africanistes, t.32, 275-323. 